Mens

revue d'histoire intellectuelle de l'Amérique française

\title{
Trois collections de livres illustrés des Éditions Albert Lévesque (1926-1937) : un cas de transferts culturels France-Québec?
}

\section{Stéphanie Danaux}

Volume 5, numéro 2, printemps 2005

L'histoire du livre au Québec, de la Nouvelle-France au XX ${ }^{\mathrm{e}}$ siècle

URI : https://id.erudit.org/iderudit/1024360ar

DOI : https://doi.org/10.7202/1024360ar

Aller au sommaire du numéro

Éditeur(s)

Centre de recherche en civilisation canadienne-française

ISSN

1492-8647 (imprimé)

1927-9299 (numérique)

Découvrir la revue

Citer cet article

Danaux, S. (2005). Trois collections de livres illustrés des Éditions Albert Lévesque (1926-1937) : un cas de transferts culturels France-Québec ? Mens, 5(2), 397-430. https://doi.org/10.7202/1024360ar
Résumé de l'article

S'il est admis que le livre illustré au Québec est l'héritier de la tradition française, de nombreuses interrogations demeurent quant à l'origine, au mode de diffusion et à l'expression de cette pénétration en terre nord-américaine. Grâce à l'exemple de trois collections de livres illustrés publiés aux Éditions Albert Lévesque dans les années 1920 et 1930, il est possible de mettre à jour une série de transferts culturels entre la France et le Québec tant dans le contenu que dans la mise en forme des ouvrages. Ces liens révèlent le rôle majeur de la médiation éditoriale ainsi que l'influence de la formation des artistes dans le développement du livre illustré au Québec. 


\title{
TROIS COLLECTIONS DE LIVRES ILLUSTRÉS DES ÉDITIONS ALBERT LÉVESQUE (1926-1937) : UN CAS DE TRANSFERTS CULTURELS FRANCE-QUÉBEC ?
}

\author{
Stéphanie Danaux \\ Département d'histoire de l'art \\ Université de Montréal/Université de Poitiers \\ stephdanaux@yahoo.fr
}

\section{Résumé}

S'il est admis que le livre illustré au Québec est l'héritier de la tradition française, de nombreuses interrogations demeurent quant à l'origine, au mode de diffusion et à l'expression de cette pénétration en terre nord-américaine. Grâce à l'exemple de trois collections de livres illustrés publiés aux Éditions Albert Lévesque dans les années 1920 et 1930, il est possible de mettre à jour une série de transferts culturels entre la France et le Québec tant dans le contenu que dans la mise en forme des ouvrages. Ces liens révèlent le rôle majeur de la médiation éditoriale ainsi que l'influence de la formation des artistes dans le développement du livre illustré au Québec.

\section{Abstract}

Though it is generally assumed that Quebec's illustrated books owe much to the French publishing tradition, little is known about how this cultural transfer took place. However, by examining three collections of illustrated books published during the interwar years by the Éditions Albert Lévesque, it is possible shed 
some light on the cultural transfers which occurred between Quebec and France regarding both the content and the formatting of books. This trans-Atlantic kinship reveals the importance of editorial mediation and of artists' training in the development of illustrated books in Quebec.

Du 16 septembre au 13 novembre 1983, l'historien de l'art Jean-René Ostiguy présente une exposition intitulée «Le livre illustré au Québec et en France (1900-1950) » à l'ancienne Galerie nationale du Canada, rebaptisée en $1984 \mathrm{Mu}-$ sée des Beaux-Arts du Canada à Ottawa. Conservateur chargé de la recherche en art canadien, Ostiguy a constaté l'apport social et culturel des livres illustrés populaires vendus à prix modique pendant la première moitié du $\mathrm{XX}^{\mathrm{e}}$ siècle et leur a consacré plusieurs années d'étude. Les premiers résultats de ses travaux, présentés la même année que l'exposition dans une brochure faisant office de catalogue ${ }^{1}$, sont organisés autour de l'hypothèse suivante : en associant l'analyse des ouvrages illustrés importés de France à l'étude de ceux qui furent ornés par les artistes québécois, il devient possible d'évaluer une manifestation de l'influence artistique française en Amérique du Nord tout en mesurant la contribution du Québec au patrimoine du livre illustré du Canada. L'exposition réunissait vingt-cinq livres québécois jumelés à autant d'ouvrages français, ce qui permettait de déployer une sélection d'illustrations favorisant la mise en relief d'une série de liens à la fois matériels, stylistiques et iconographiques. Le livre illustré au Québec est en effet le fruit de deux grandes traditions : l'anglaise $^{2}$ et surtout la française, plus évidente. Si les historiens de l'art québécois s'accordent pour reconnaître l'existence d'une filiation française dans le contenu et le style des images, cette remarque semble également pouvoir s'appliquer aux formats, papiers, reliures, mises en page et prix de nombreuses éditions jusqu'au milieu du $\mathrm{XX}^{\mathrm{e}}$ siècle. Bien que ces hy- 
pothèses aient été posées depuis plus de vingt ans, aucune étude n'a approfondi ce constat. Nous postulons donc que la production de livres illustrés publiés au Québec au cours des années 1920 et 1930 est le résultat d'un déplacement et d'une appropriation de problématiques de recherche, de pratiques et de mouvements culturels - simultanément artistiques, littéraires, intellectuels et éditoriaux — souvent empruntés à l'Europe, et plus spécifiquement à la France. Toutefois, cette influence française demeure bien souvent malaisée à identifier, à délimiter et à expliquer. Ces manifestations de transferts culturels, favorisés par des échanges nombreux et variés, laissent en effet peu d'indices sur les liens établis entre les agents des champs de production québécois et français ainsi que sur la manière dont chaque intermédiaire a contribué au phénomène. Il s'agit donc de répondre à plusieurs interrogations quant à l'origine, au mode de diffusion et à l'expression de cette pénétration française en terre nord-américaine.

Certains ouvrages illustrés des Éditions Albert Lévesque semblent représentatifs de cette situation. De janvier 1926 à mars 1937, le jeune Albert Lévesque (1900-1979) dirige sa propre maison d'édition et gère la Librairie de l'Action canadienne-française dont il a acquis le fonds de commerce. Avec quelques autres éditeurs de son temps, il joue un rôle majeur dans le développement du champ éditorial québécois de la fin des années 1920 et du début des années 1930. Cet article se propose d'analyser trois collections de livres illustrés publiés aux Éditions Albert Lévesque au cours de cette période. Ces trois ensembles se caractérisent par des contenus textuels distincts. La collection Les Romans historiques, issue du catalogue de l'Action canadienne-française, regroupe onze récits pour adolescents, tous illustrés. La série Les Poèmes, fondée en 1928, réunit treize titres dont cinq illustrés. Enfin, Les Romans de la 
jeune génération sont créés en 1931 et réunissent quatre romans psychologiques dont trois sont accompagnés de bois gravés. Pour déceler, expliquer et tenter de retracer l'origine d'éventuels signes de transferts culturels, nous tenterons tout d'abord de situer la trajectoire d'Albert Lévesque au sein du milieu éditorial québécois des années 1920 et 1930 . Nous insisterons ensuite sur les caractéristiques textuelles et formelles des filiations révélées au sein des livres illustrés diffusés sous sa direction. Nous nous attacherons aux choix des textes et à l'ensemble de la mise en pages, à travers les illustrations (hors et in-texte) et les diverses ornementations (vignettes, bandeaux, en-têtes, culs-de-lampe ${ }^{3}$ ), en basant notre analyse sur une comparaison avec deux collections françaises diffusées au cours de la même période : Le Livre de demain des Éditions Arthème Fayard et Cie et Le Livre moderne illustré des Éditions Joseph Ferenczi et Fils. Tous les éléments d'une publication sont en effet tributaires du projet éditorial ainsi que du rapport qui s'établit entre les différents médiateurs de l'œuvre que sont l'éditeur, l'illustrateur, l'écrivain (que ce dernier soit vivant ou non), le typographe, l'imprimeur, le relieur, le metteur en page, etc.

\section{Naissance du champ éditorial québécois}

L'univers du livre au Québec est longtemps demeuré restreint : de 1764 à 1820, moins de 1200 imprimés ont été répertoriés toutes catégories confondues, à l'exception des journaux. Or, plus de $50 \%$ de cette production fait moins de quatre pages tandis que les livres au sens propre sont essentiellement constitués d'ouvrages pratiques et religieux ainsi que de quelques contes et romans du terroir, dont seulement $25 \%$ fait plus de cinquante pages ${ }^{4}$. En raison de la communauté de langue, c'est d'Europe ${ }^{5}$, et plus spécifiquement de France, que la province importe l'ensemble du matériel di- 
dactique et littéraire nécessaire, agrémenté d'images ou non ${ }^{6}$. Selon François Landry, la production québécoise correspond à moins de $5 \%$ des besoins du lectorat. Elle est supplétive et répond à des nécessités locales, couvrant les secteurs qui ne sont pas pris en charge par l'édition étrangère tandis que l'impression se consacre principalement aux journaux et aux revues. L'historien Jacques Michon estime que ces chiffres restent applicables au moins jusqu'en $1859^{7}$. C'est en effet à cette époque que les politiques scolaires du Dr J.-B. Meilleur, Surintendant de l'Instruction Publique, et de son successeur en 1855, Pierre-Joseph-Olivier Chauveau, donnent leurs premiers résultats. Inférieur à $30 \%$ pour l'ensemble de la province (dont $50 \%$ pour la seule ville de Québec), le taux d'alphabétisation croît ensuite de décennie en décennie pour atteindre 74,4\% des Québécois entre 1890 et $1899^{8}$, phénomène coïncidant avec les progrès de l'urbanisation'. Après 1900, l'instruction touche plus de $90 \%$ de la population : l'ensemble de la nation a désormais accès à l'éducation et à la lecture. Cet essor du lectorat au sein des classes populaires constitue la condition nécessaire à une extension de la consommation du livre, suscitant à son tour l'éclosion d'un champ éditorial plus diversifié. Le livre n'est plus réservé aux classes dominantes. La hausse de la production des manuels scolaires et des ouvrages littéraires ${ }^{10}$ ainsi que la multiplication des librairies, particulièrement à Montréal, sont significatives de cette évolution.

Dans le secteur plus restreint du livre illustré québécois, le $\mathrm{XIX}^{\mathrm{e}}$ siècle constitue une période peu connue. Parmi les titres édités au Québec, le récit historique est alors le genre dominant. Il prend le plus souvent la forme de traités de géographie valorisant les régions du Canada et de biographies apologétiques des figures politiques ou religieuses de la province. Leur fonction est avant tout éducative. Quant au nombre de publications littéraires illustrées au cours de cette période, il s'élève à une quinzaine, en majorité des recueils de 
poésie $^{11}$. Silvie Bernier a remarqué que leur contenu n'est pas spécifiquement destiné à l'élite mais à l'ensemble de la population. À ce titre, ils se conforment aux règles traditionnelles de l'art populaire: les formats et matériaux demeurent modestes tandis que les illustrations sont généralement reproduites en noir et blanc, procédé moins coûteux que la couleur. Les situations manichéennes et moralisatrices décrites dans les textes, proches de la vie quotidienne, sont mises en images par une multiplicité de scènes et de portraits destinés à faciliter l'identification au héros. Le traitement des compositions se caractérise par une esthétique réaliste, car les illustrateurs obéissent aux règles du dessin académique et accordent un très faible espace à la recherche plastique ${ }^{12}$. Cette situation se maintient jusque dans les premières décennies du $\mathrm{XX}^{\mathrm{e}}$ siècle.

C'est en effet au milieu des années 1920 que la production du livre illustré québécois prend son envol. Silvie Bernier signale qu'environ deux cents titres littéraires (fiction et poésie) accompagnés d'illustrations sont édités avant les années $1940^{13}$. Cet essor coïncide avec l'arrivée dans le champ éditorial naissant de plusieurs éditeurs indépendants, c'est-àdire séparés de l'imprimerie, de la librairie ou de la presse : il s'agit d'Eugène Achard, de Louis Carrier, d'Albert Pelletier, d'Édouard Garand et surtout d'Albert Lévesque. Pour ces jeunes hommes, "il s'agit de doter le Canada français de maisons de littérature générale capables de combler les besoins du milieu et susceptibles de concurrencer les sociétés européennes sur le marché local ${ }^{14}$ ». Avec cette génération, «le nouvel éditeur apparaît dès lors comme le représentant le plus légitime de la communauté littéraire, le médiateur idéal et le promoteur par excellence de la création artistique ${ }^{15} \%$. Il participe en effet à tous les stades de l'élaboration du livre, illustré ou non. 
Titulaire d'un baccalauréat es arts, Albert Lévesque arrive à Montréal en 1922 et s'inscrit en droit à l'Université de Montréal avant d'opter pour une formation en publicité à l'École des Hautes Études commerciales. Grâce à quelques recommandations, il établit à la même époque des contacts étroits avec la Ligue d'Action française dirigée par l'abbé Lionel Groulx (1878-1967) ${ }^{16}$. Progressivement engagé dans les affaires de la Ligue, Lévesque met à jour certaines manœuvres frauduleuses ${ }^{17}$. Afin d'étouffer le scandale et de protéger les coupables, les directeurs de la Ligue lui en confient aussitôt la gérance, mais le jeune homme découvre alors une situation financière largement déficitaire. En 1926, il décide pourtant de sauver l'entreprise d'une faillite humiliante en rachetant discrètement la maison d'édition et tout son fonds de commerce avec l'aide de son beau-père ${ }^{18}$. À la suite d'une entente avec la Ligue ${ }^{19}$, il publie sous le nom de «Bibliothèque de l'Action française » jusqu'en 1927 puis sous celui de "Librairie d'Action canadienne-française Limitée ». Au terme de l'accord en 1931, le nom des "Éditions Albert Lévesque " se répand. Si ces changements de raison sociale illustrent les mutations progressives de la direction de l'entreprise, ils révèlent également l'apparition d'une nouvelle ère au sein de la sphère éditoriale québécoise, celle de l'éditeur moderne. Après cette rupture avec la Ligue, Lévesque devient totalement indépendant : il abandonne la librairie et le commerce d'importation de livres européens pour se consacrer exclusivement au métier d'éditeur de nouveautés littéraires québécoises. Albert Lévesque a en effet rapidement pris conscience du rôle et du pouvoir de l'éditeur, qui ne peut plus être considéré comme un médiateur neutre entre l'écrivain et le public.

À la même époque, d'autres intellectuels participent au développement du champ éditorial. Dès 1928, Louis Carrier fonde à Montréal les Éditions du Mercure/The Mercury Press 
(1928-1931) consacrées à l'édition de nouveautés canadiennes en français et en anglais. L'entreprise de Carrier et celle de Lévesque sont suivies de près par les Éditions du Totem (1933-1938) d'Albert Pelletier et celles du Zodiaque (19341942) dirigées par Eugène Achard. Le catalogue des Éditions Albert Lévesque, établi par Liette Bergeron, apparait toutefois comme le plus volumineux de la période en comprenant 254 titres $^{20}$ tandis que Pierre Landry ${ }^{21}$ signale que celui du Zodiaque, en incluant tous les imprimés parus jusqu'en 1939, rééditions comprises, s'élève à vingt-quatre titres, celui du Mercure vingt-deux et celui du Totem à moins de vingt. Parmi ces quatre éditeurs, Lévesque et Carrier contribuent activement au patrimoine du livre illustré québécois : un tiers de leur catalogue respectif est en effet accompagné d'images. Sept livres illustrés sont ainsi publiés aux Éditions Louis Carrier, parmi lesquels on remarque tout particulièrement Other Days, Other Ways/Vieilles choses, vieilles gens (1928) de Georges Bouchard avec des bois gravés d'Edwin H. Holgate (18921977) ainsi que Le Manoir banté (1928) de Régis Roy et La Pension Leblanc (1927) de Robert Choquette, ornés respectivement de dix et cinq dessins de Jean-Paul Lemieux (19041990).

\section{Le livre illustré français au Québec}

Parallèlement à cette évolution, l'édition française continue d'alimenter le marché québécois. L'installation en 1894 et 1896 de deux libraires indépendants dans le Quartier latin de Montréal, Jules A. Pony et Cornélius Déom ${ }^{22}$, a largement contribué au phénomène. Moins liées au secteur scolaire et fréquentées principalement par des acheteurs individuels, ces boutiques sont définies par Lucie Robert comme «les seules vraies librairies ${ }^{23}$ » de la période. Si les goûts des lecteurs et bibliophiles de l'époque sont peu connus, Déom et Pony ont 
toutefois été, jusqu'au milieu du $\mathrm{XX}^{\mathrm{e}}$ siècle, un important facteur de diffusion de romans illustrés à prix modiques provenant d'éditeurs parisiens. Ces ouvrages semblent avoir constitué une source d'inspiration, d'une part, pour les éditeurs et, d'autre part, pour les artistes québécois. Ostiguy fut le premier à percevoir l'influence de ces éditions étrangères négligées par le public contemporain et les professionnels du livre à cause de leur prix bon marché ${ }^{24}$. Il s'agit de La Modern Bibliothèque (1904-1923), publiée par les Éditions Arthème Fayard et Cie et dont les titres sont ornés de similigravures, à laquelle succède Le Livre de demain (1923-1946). Inspirées par le succès de leurs concurrents, les Éditions Joseph Ferenczi et Fils publient et exportent à leur tour les nombreux titres de la série Le Livre moderne illustré (1924-1939). Issue d'un souci de démocratisation de la culture, cette production intermédiaire entre l'édition littéraire et l'édition populaire constitue en France une formule nouvelle: les tirages importants (entre 40000 et 50000 exemplaires) $)^{25}$ et le prix abordable sont pour la première fois compatibles avec une véritable recherche de qualité tant dans la forme que dans le contenu. Ces collections sont composées de romans récents, généralement des classiques de la littérature française ou des succès de librairie, et sont toutes illustrées de gravures sur bois ou de similigravures dont certaines en couleurs. On ne peut cependant en aucun cas parler de publications de luxe. Les formats demeurent modestes et se veulent pratiques, contrairement à de nombreux livres de peintre ou d'artiste qui nécessitent souvent un rituel respectueux dans la manipulation. Les couvertures ne sont pas rigides et le papier, de qualité moyenne, vieillit assez mal ${ }^{26}$. Enfin, les prix demeurent modiques : seulement 95 centimes pour La Modern Bibliothèque puis entre deux et cing francs pour Le Livre de demain et Le Livre moderne illustré. Antérieures ou parallèles à celles qui voient le jour au Québec au cours des années 1920 et 1930, ces collections 
françaises sont abondamment distribuées dans la province. Ostiguy signale d'ailleurs leur présence dans de nombreuses bibliothèques personnelles ou publiques des années 1920 à 1940 et même dans les restaurants-librairies et librairies ventes-échanges où chaque livre est estampillée $e^{27}$.

\section{Importance du contenu dans Les Romans historiques, Les Romans de la jeune génération et Les Poèmes}

Bien que Les Romans de la jeune génération, Les Poèmes et Les Romans historiques des Éditions Albert Lévesque soient considérés par Jean-René Ostiguy comme «les principaux parallèles des collections française ${ }^{28} "$, l'influence des ouvrages de Fayard et de Ferenczi sur les éditeurs, les artistes et le public québécois demeure difficile à circonscrire. À l'instar des dirigeants de Fayard et de Ferenczi, Albert Lévesque se donne pour mission dès 1926 de publier des textes de qualité, œuvres d'écrivains canadiens-français contemporains, généralement laurentiens, jeunes ou reconnus, le tout à un prix abordable (entre 0,75 et 1 dollar). À une époque où l'essentiel du répertoire national destiné à la jeunesse date du XIX ${ }^{\mathrm{e}}$ siècle, il n'hésite pas à diffuser des textes nouveaux et encourage même ses contemporains à écrire spécialement pour le jeune public ${ }^{29}$. C'est ainsi que parmi les auteurs des titres de la collection Les Romans historiques, Albert Lévesque recrute des écrivains déjà primés comme Harry Bernard ${ }^{30}$ et MarieAntoinette Grégoire-Coupa ${ }^{31}$, respectivement auteurs de Montcalm se fâche (1935), accompagné de linogravures d'Henri Beaulac (1914-1994) (Illustration 1), et de La Sorcière de l'́lot noir (1935), illustré de dessins par Jean Palardy (1905-1991). Lévesque enrôle également de nouveaux auteurs très prolifiques comme Maxine ${ }^{32}$ dont six titres trouvent place dans Les Romans historiques parmi lesquels Le petit Page de Frontenac (1935) illustré par Jean-Paul Lemieux (Illustration 2$)^{33}$. Avec ses épo- 


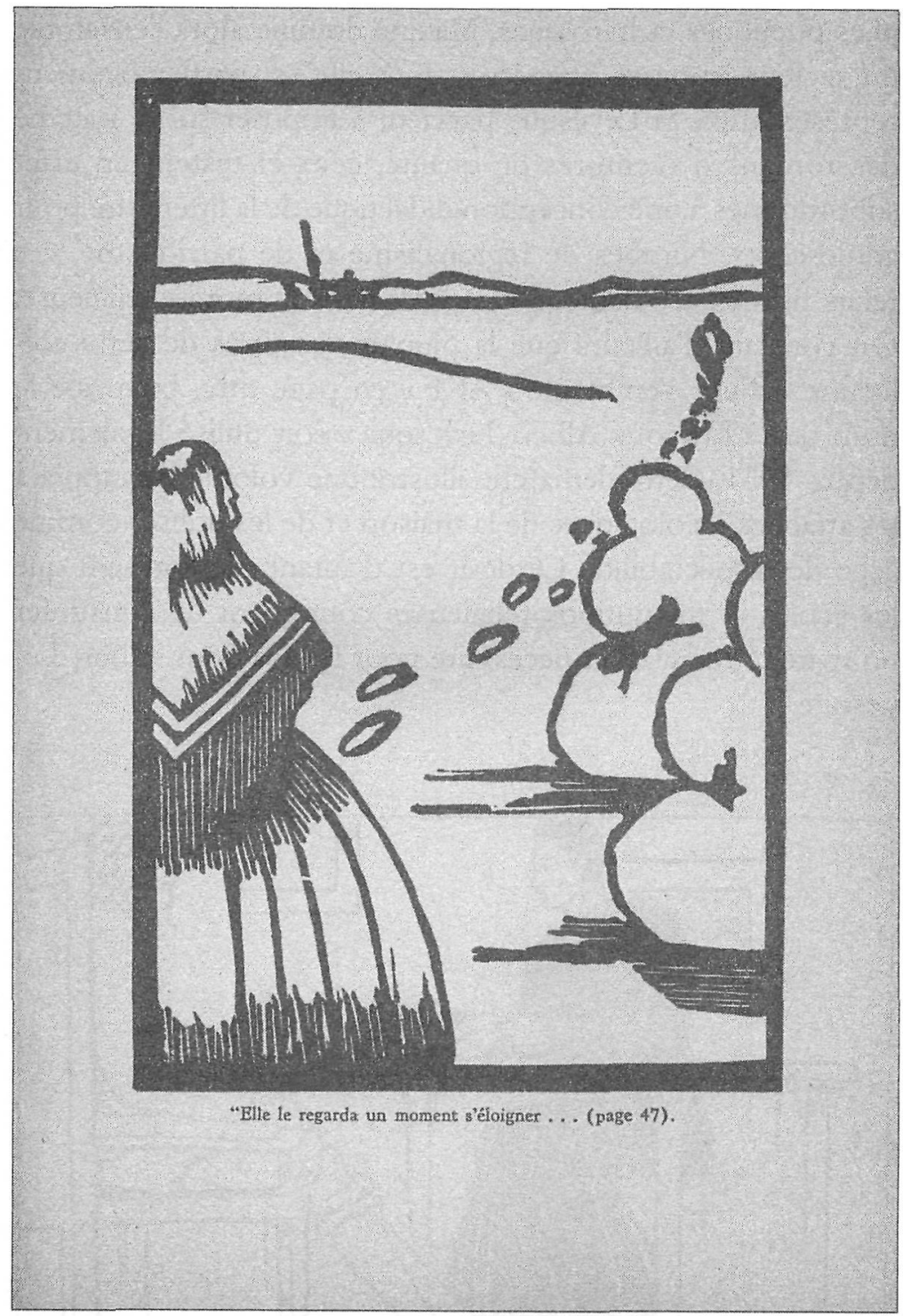

Illustration 1 : Harry Bernard, Montcalm se fâche, linogravure en hors-texte d'Henri Beaulac, Montréal, Albert Lévesque, 1935 (Coll. « Romans historiques »), 149 p., 24 cm, Bibliothèque nationale du Québec. 
pées poétiques et héroïques, Maxine domine alors l'ensemble du secteur jeunesse québécois dont elle est particulièrement représentative. Si Lévesque parvient à imposer sur le marché des romans d'aventures de qualité, ceux-ci restent en effet subordonnés à une conception didactique de la littérature pour adolescents. Nimbés de régionalisme et de patriotisme, ces récits historiques demeurent ancrés dans la morale religieuse. On constate d'ailleurs que la plupart des titres de cette collection affiche l'emblème " $\mathrm{ACF}$ » en page titre, bien que le nom des "Éditions Albert Lévesque » soit utilisé légalement depuis 1931. Cette démarche illustre une volonté de rappeler les attaches idéologiques de la maison et de les utiliser comme gage de respectabilité. Ce désir est d'autant plus présent que les écoles et institutions religieuses continuent de constituer un marché rentable et nécessaire pour les Éditions Albert Lévesque $^{34}$.

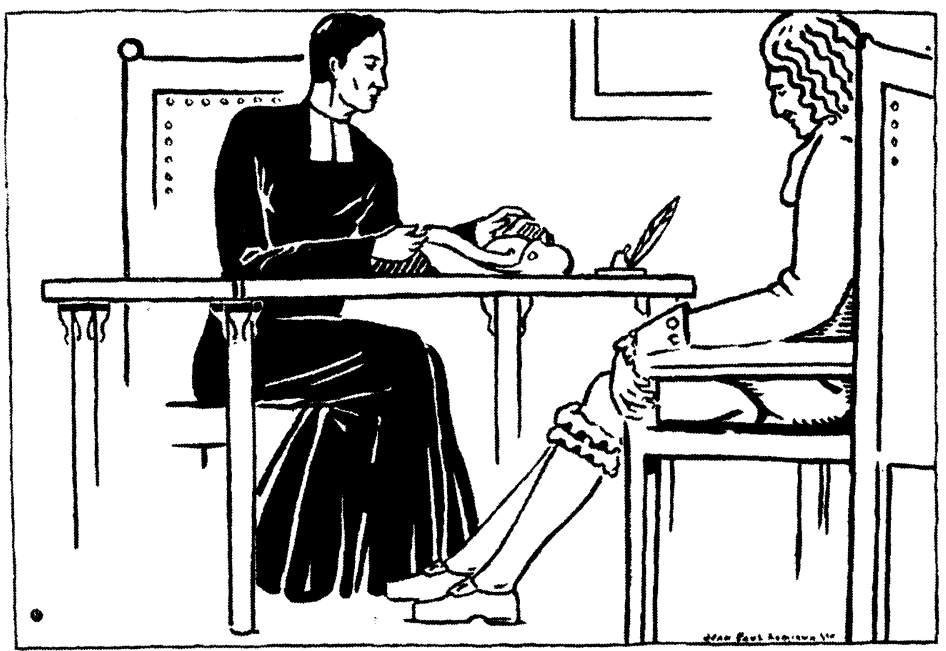

Illustration 2 : Maxine, Le petit Page de Frontenac, double-page avec un dessin hors-texte de Jean-Paul Lemieux, Montréal, Albert Lévesque, 1935 (Coll. «Romans historiques »), 162 p, $24 \mathrm{~cm}$, Bibliothèque nationale du Québec. 
Lévesque semble toutefois soucieux de s'affranchir de la pression cléricale. Si Les Romans bistoriques sont d'une moralité irréprochable, la publication d'une nouvelle collection très audacieuse suscite la polémique et nous éclaire sur la prise de distance du jeune éditeur avec le nationalisme conservateur de ses prédécesseurs. Le lancement en 1931 de la série Les Romans de la jeune génération coïncide en effet avec le moment où la Ligue perd son droit de regard sur la production et où Lévesque commence à faire paraitre des ouvrages sous son propre nom. Il s'agit des ouvrages La Chair décevante de Jovette-Alice Bernier avec un frontispice gravé sur bois par Alyne Gauthier (1908-1955), Dans les Ombres d'Éva Senécal et Le Dilettante de Claude Robillard, chacun illustré avec cinq bois originaux de Jean-Paul Audet (Illustration 3), ainsi que L'Initiatrice de Rex Desmarchais, non illustré. Ces quatre ouvrages aux titres accrocheurs sont des récits psychologiques tournés "vers l'exploration des mœurs citadines ${ }^{35}$ ", genre montant au détriment du roman du terroir. Ils marquent une rupture dans le milieu littéraire québécois et ne sont pas sans provoquer quelques remous. La collection est d'ailleurs interrompue après seulement quatre titres sans aucune justification, même s'il semble raisonnable de supposer que l'éditeur, très prudent, redoutait d'irriter ses commanditaires des milieux scolaire et religieux. En favorisant ainsi de jeunes écrivains, il avait pourtant rejoint les préoccupations de Fayard et de Ferenczi : proposer des récits à la fois divertissants et proches des préoccupations contemporaines tout en visant un nouveau lectorat, le grand public, composé d'adultes qui lisent pour se divertir et s'alimentent en romans français récents chez les libraires Déom et Pony.

Enfin, la seule collection de poésie publiée aux Éditions Albert Lévesque, intitulée Les Poèmes, compte cinq titres illustrés: Les Masques déchirés (1932) de Jovette-Alice 


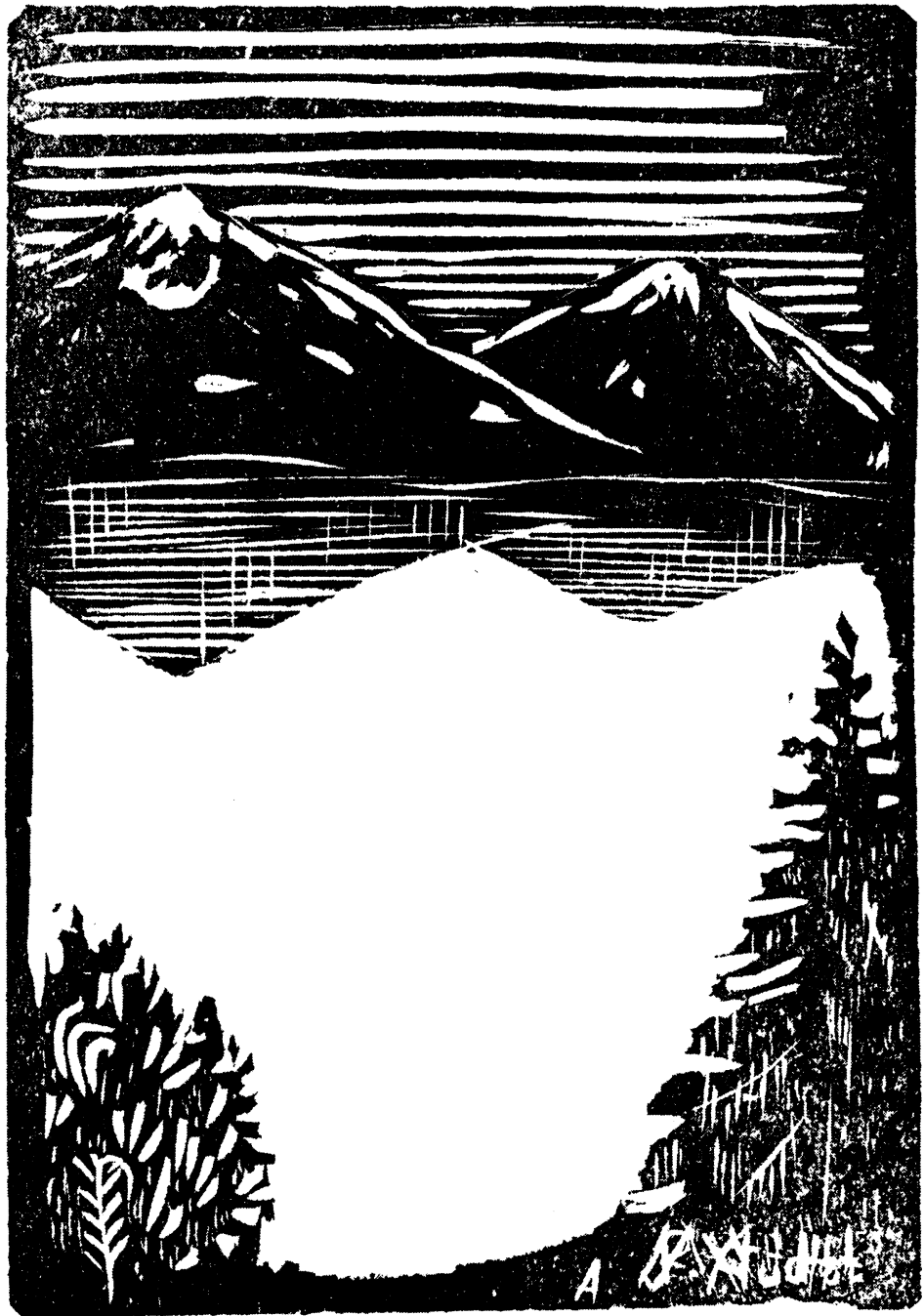

Illustration 3 : Éva Senécal, Dans les Ombres, double-page avec une gravure sur bois en hors-texte de Jean-Paul Audet, Montréal, Albert Lévesque, 1931 (Coll. «Les Romans de la jeune génération »), 153 p., 19 cm, Bibliothèque nationale du Québec. 


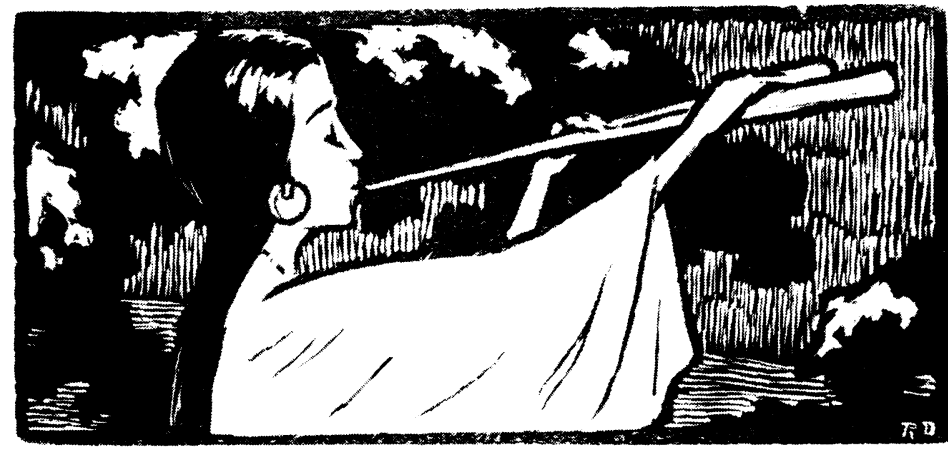

Illustration 4 : Ulric Gingras, Du Soleil sur l'étang noir, avec une vignette en tête de chapitre gravée sur bois de Rodolphe Duguay, Montréal, Albert Lévesque, 1933 (Coll. «Les Poèmes »), 187 p., $20 \mathrm{~cm}$, Bibliothèque nationale du Québec.

Bernier illustré par Robert La Palme (1908-1997), Quand j'parl'tout seul (1932) de Jean Narrache (pseudonyme d'Émile Coderre) avec dix dessins de Jean Palardy, Dominantes (1933) de René Chopin avec quatre fusains d'Adrien Hébert (18901967), Du Soleil sur l'étang noir (1933) d'Ulric Gingras avec des bois gravés originaux de Rodolphe Duguay (1891-1973) (Illustration 4) et enfin Aux Sources claires (1935) de Jacqueline Francour avec des gravures sur linoléum de Simone Hudon (1905-1984) (Illustration 5). L'historien Jacques Michon souligne que cette série semble être le résultat d'une action éditoriale moins concertée $e^{36}$, car on ne constate de réelle unité ni sur le plan du contenu ni sur celui de la présentation matérielle. La jeune génération, incarnée par Jovette-Alice Bernier ou Jean Narrache ${ }^{37}$, côtoie les auteurs plus traditionnels comme René Chopin. Quelques valeurs sûres ou primées suffisent ainsi à conférer à l'ensemble le crédit nécessaire pour rassurer le lecteur dans son choix. On peut deviner dans cette diversification de la production une volonté d'élargir le lectorat. Dans le même temps, on constate que seulement cinq des treize titres de la série sont illustrés tandis que les formats, les 


\section{JACQUELINE FRANCOEUR}

\section{AUX \\ SOURCES CLAIRES}

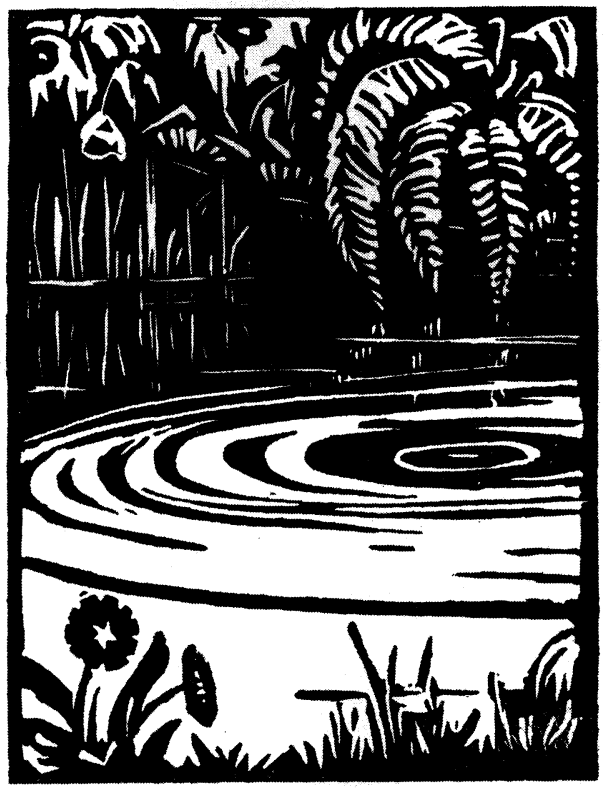

ILLUSTRATIONS DE SIMONE HUDON

ÉDITIONS ALBERT LÉVESQUE MONTRÉAL, 1935

Illustration 5 : Jacqueline Francœur, Aux Sources claires, couverture avec une vignette gravée sur linoléum de Simone Hudon, Montréal, Albert Lévesque, 1935 (Coll. «Les Poèmes »), 151 p., 19 cm, Université du Québec à Montréal. 
couvertures, la mise en pages et les techniques d'illustration ne sont pas toujours identiques, ce qui est contraire au principe de collection. Le nom de cette série est parfois même absent des exemplaires, comme dans Aux Sources claires de Jacqueline Francœur, ce qui risque de rendre difficile au lecteur l'attribution à la collection. La présence ponctuelle d'un sigle (une lyre entremêlée aux initiales de l'ACF) associée à un état des publications situé sur le plat verso des ouvrages ${ }^{38}$ semble suffire à la réunion de ces treize titres sous le label «collection ", donnant ainsi du sens à un ensemble pourtant caractérisé par son manque d'homogénéité. Comme le souligne Gérard Genette, la notion récente de collection est si puissante que son absence «est ressentie par le public et articulée par les médias comme une sorte de collection implicite ou a contrario ». L'absence d'indication devient une dénomination officieuse. Genette ajoute que «le label de la collection, même sous cette forme muette, est donc un redoublement du label éditorial, qui indique immédiatement au lecteur potentiel à quel type, sinon à quel genre d'ouvrage il a affaire ${ }^{39} »$. Il s'agit ainsi d'une approche résolument moderne dont l'objectif est de canaliser un public cible autour de quelques titres regroupés en collection. Ce procédé permet de fidéliser le lecteur et de développer une régularité d'achat en suscitant de nouveaux besoins auxquels l'éditeur se propose de répondre. Le principe de collection est « une façon pour l'éditeur littéraire, qui pratique une édition non-programmée, d'introduire dans son mode de fonctionnement une forme de programmation ${ }^{40} »$.

\section{Une conception esthétisante du livre}

D'un point de vue formel, tous ces ouvrages se définissent par une bonne qualité technique. Cette caractéristique est clairement revendiquée par les collections Le Livre de demain et Le Livre moderne illustré, le souci du beau livre à la 
française étant une tradition développée par l'Art Nouveau en Europe au début du $\mathrm{XX}^{\mathrm{e}}$ siècle. Les propriétés physiques communes sont nombreuses. Les formats sont proches, les hauteurs variant d'une vingtaine de centimètres pour Les Romans de la jeune génération et Le Livre moderne illustré à vingtquatre centimètres pour Le Livre de demain et Les Romans bistoriques. Les mises en page sont aérées avec des marges larges et une justification du texte allant jusqu'au deux-tiers du format. Les caractères typographiques, qui se détachent clairement de la page, sont statiques, sobres et lisibles tout en étant souples et élégants. L'impression des illustrations comme celle du texte est bien contrastée, quoique parfois imprécise chez Ferenczi. L'usage du bandeau ou de la vignette en tête de chapitre est généralisé ainsi que celui des culs-de-lampe. Enfin, l'usage de la gravure sur bois comme technique d'illustration est largement privilégié. Si son recours est exclusif dans Le Livre moderne illustré et Le Livre de demain, cette remarque s'applique à la moitié des ouvrages illustrés des collections Les Poèmes et Les Romans historiques ainsi qu'à tous Les Romans de la jeune génération. La beauté des contrastes des blancs et des noirs se marie en effet harmonieusement avec les caractères typographiques et contribue à accorder à chaque ouvrage une élégance mesurée.

Ces constats peuvent sembler étonnants à une époque où la gravure constitue une pratique peu répandue chez les artistes et méconnue du public québécois. En effet, il n'existe alors aucun enseignement de la gravure, « en dehors de la formation dispensée par les ateliers de lithographie et de photogravure commerciale qui alimentent les journaux illustrés ${ }^{41}$ ». Nombreux sont donc les artistes à rapporter d'Europe cette nouvelle sensibilité envers l'estampe. Rodolphe Duguay ${ }^{42}$ est ainsi présent à Paris de 1920 à 1927 tandis qu'Edwin H. Holgate $^{43}$ y effectue deux séjours, d'abord de 1912 à 1914 
puis de 1920 et 1922 . Ils fréquentent les académies de la Grande Chaumière, Julian, Colarossi ou l'École des beauxarts de Paris dans lesquelles ils s'exercent inlassablement aux subtilités du dessin. C'est néanmoins en autodidactes, au début des années 1920, que ces deux futurs peintres et illustrateurs se forment aux techniques modernes de l'eau-forte et de la gravure sur bois, au gré de leurs rencontres et de leurs lectures. On sait par exemple que Duguay est initié au bois en 1924 par son ami le peintre-graveur Paul-Albert Moras ${ }^{44}$ et par son compatriote Louis-Philippe Beaudoin, alors étudiant en reliure à l'École Estienne de Paris. Selon Denis Martin, c'est d'ailleurs Beaudoin qui attira le premier "son attention sur le renouveau du bois gravé d'illustration en France ${ }^{45}$ ». Ce médium connaît alors en France une renaissance spectaculaire dont les plus célèbres réussites dans le milieu de l'édition littéraire populaire sont précisément les collections Le Livre de demain (1923) et Le Livre moderne illustré (1924). On sait également que Duguay se procure, à la même époque, trois ouvrages qui vont orienter sa production: Les Décorateurs du livre (1922) de Charles Saunier, La Technique moderne du bois gravé (1925) de Maurice Busset et Le Manuel de gravure sur bois (1926) de Morin-Jean ${ }^{46}$, graveur fameux qui réalise à la même époque 36 bois originaux pour le roman de Pierre Louÿs, Aphrodite. Mcurs antiques, dans la série Le Livre de demain (1927).

À leur retour au Canada, ce nouvel attrait pour le bois se développe tout particulièrement dans le domaine du livre illustré, si bien que Denise A. Ostiguy considère que les artistes québécois « continuent la pensée d'Auguste Lepère (18491918), graveur français qui disait que la façon la plus noble d'illustrer un livre demeure la gravure sur bois ${ }^{47} »$. Ils diffusent ensuite leurs connaissances par leur pratique - comme en 1924 lors de la première exposition de gravures sur bois réunissant les œuvres d'Edwin H. Holgate, d'Ivan Jobin (1885- 
1975) et de Maurice LeBel (1898-1963) à l'Art Association de Montréal - ou par leur enseignement. Le professorat devient en effet le moyen privilégié pour développer ce médium au Québec, principalement grâce à la fondation en 1921 et 1922 des deux grandes écoles de beaux-arts de Québec et de Montréal. C'est d'ailleurs Holgate, bientôt célèbre dans le milieu de l'illustration pour ses bois gravés dans Vieilles choses, vieilles gens de Georges Bouchard et Metropolitan Museum de Robert Choquette $^{48}$, qui devient en 1926 le premier professeur de gravure sur bois de l'École de Montréal ${ }^{49}$. Alyne Gauthier, également illustratrice, occupera ce poste à partir de 1941. À l'École des beaux-arts de Québec, Simone Hudon succède à Robert W. Pilot (1898-1967) ${ }^{50}$ de 1931 et 1945 au poste de professeur de gravure, après avoir été son élève pendant cinq ans. Ces deux écoles constituent des lieux de professionnalisation de la fonction artistique et si l'enseignement de la lithographie semble plutôt se rattacher à la conception et à l'impression d'affiches publicitaires, celui de la gravure sur bois est directement lié au monde du livre et de son illustration. Ces deux écoles étant créées à une époque où le renouveau du livre illustré atteint son apogée en Europe, les professeurs ne manquent pas de faire valoir à leurs élèves les possibilités de débouchés professionnels dans cette industrie ${ }^{51}$. C'est ainsi qu'ils contribuent à l'essor général du livre illustré au Québec à partir du milieu des années 1920 et à la formation d'une nouvelle génération d'illustrateurs québécois qui, comme Henri Beaulac, se distingue de la précédente par l'utilisation privilégiée de la gravure sur bois ou sur linoléum. Les éditions de Fayard et de Ferenczi marquent toutefois « les derniers feux de la gravure sur bois dans l'édition à grand tirage ${ }^{52}$ » et on constate, à partir des années 1940, que l'usage de l'illustration du livre disparaît peu à peu. 


\section{La mise en pages : positionnement du texte, des illustrations et des éléments de décoration}

Si la quantité des illustrations est toujours supérieure à vingt dans les collections de Fayard et de Ferenczi, elle varie chez Lévesque de une (le frontispice de La Chair décevante) à une douzaine (Aux Sources claires ou tous Les Romans historiques $)^{53}$. Il est vrai que le coût des illustrations est plus facilement défrayé pour Fayard et Ferenczi dont les tirages sont très élevés (entre 40000 et 50000 exemplaires) tandis que ceux de Lévesque tournent généralement autour des 1000 exemplaires ${ }^{54}$, voire même moins pour l'édition de poésie. Dans toutes ces éditions, on constate toutefois un souci commun de valorisation de l'image d'où résulte un certain manque d'intégration entre le contenu narratif et le contenu plastique. La filiation France-Québec est ici manifeste. En évoquant diverses publications illustrées au Québec au cours des années 1900 et 1910, Pierre Landry remarquait déjà que : «ce conservatisme découle sans doute de l'affiliation de l'édition québécoise à l'édition française qui, elle aussi, malgré une certaine forme de livre artistique destinée aux bibliophiles, perpétue un conservatisme peu en accord avec l'évolution générale des arts décoratifs ${ }^{55}$ ». Jusque dans les années 1940 en France, l'organisation de la typographie et la mise en pages des illustrations s'effectuent en effet dans une tradition de stricte séparation entre le texte et les images. Suivant leurs dimensions, les images sont placées selon une disposition orthogonale, sur toute la justification ou proportionnellement à celle-ci, généralement selon la règle de la symétrie la plus rigoureuse. On constate que cet usage s'applique tout spécialement «dans les livres scolaires ou dans les collections populaires illustrées de bois gravés qui paraissent entre les deux guerres et dont les archétypes sont "Le Livre de demain" (Fayard) et "Le Livre moderne illustré" (J. Ferenczi $)^{56} »$. Cha- 
cun des ouvrages issus de ces collections est en effet agrémenté d'un frontispice hors-texte précédant (chez Fayard) ou suivant (chez Ferenczi) une page-titre illustrée, puis de nombreuses vignettes systématiquement placées en tête et fin de chapitre mais rarement intercalées dans le corps de texte (Illustration 6$)^{57}$. Le phénomène se vérifie également chez les imprimeurs québécois : toutes les illustrations insérées dans Les Romans de la jeune génération et dans Les Romans bistoriques sont des hors-texte. Si l'explication peut en être d'ordre technique (le hors-texte est plus facile à mettre en œuvre, donc plus rapide et moins onéreux), il semble que les typographes québécois, à l'instar de leurs collègues français de la première moitié du $\mathrm{XX}^{\mathrm{e}}$ siècle, "ont très profondément intégré cette disjonction de l'image et du texte ${ }^{58} »$. En revanche, la collection Les Poèmes est la seule à présenter soit des hors-texte (tous les dessins de Palardy pour Quand j'marche tout seul), soit des vignettes en tête et fin de chapitres (les gravures sur linoléum de Hudon pour Aux Sources claires), soit une mise en pages variée qui alterne les hors et les in-texte (les quatre dessins de Hébert pour Dominantes), le tout dans le respect de la symétrie de la page et dans les limites de la justification du texte. Dans Les Romans bistoriques, on constate également la présence occasionnelle de bandeaux (avec une succession de motifs palmés très stylisés) situés en tête de chapitre ainsi que celle de culs-de-lampe (petits carrés à motif palmé correspondant à une unité d'un bandeau) dont la disposition obéit aux mêmes règles. Cette quête d'équilibre, qui relève de la tradition typographique française, favorise à chaque fois la création d'un ensemble harmonieux, élégant et toujours sans fioriture, l'objectif étant de proposer au lecteur un objet raffiné.

\section{Le statut des illustrateurs}

Une caractéristique importante des collections de Fayard et de Ferenczi consiste à faire apparaitre systématiquement le 


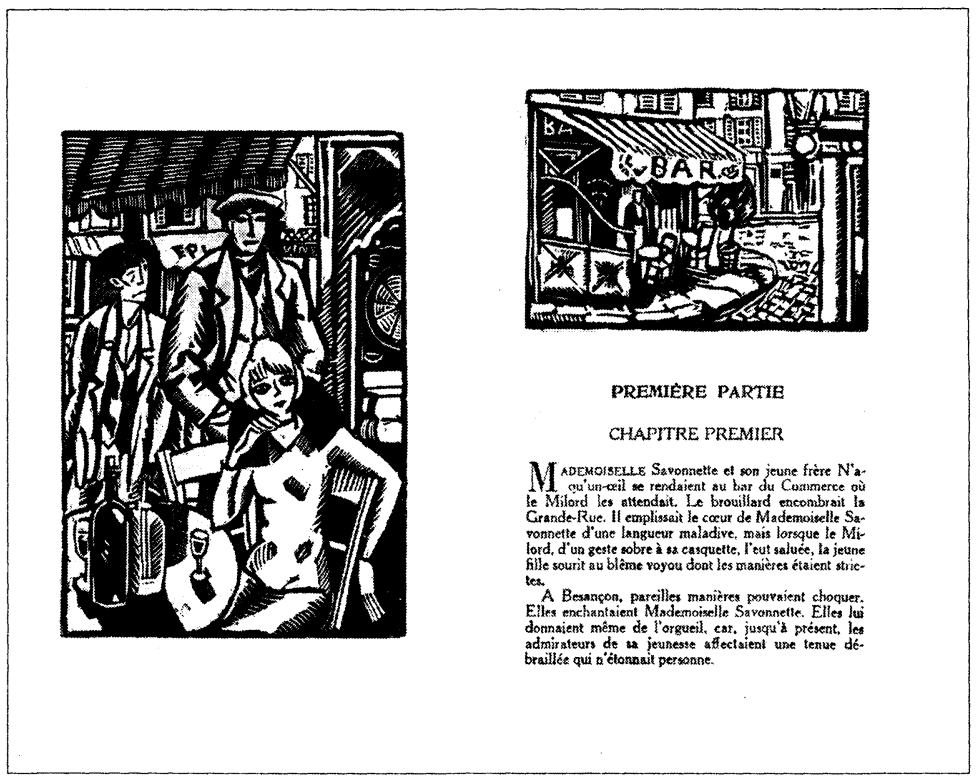

Illustration 6 : Francis Carco, Les Innocents, double-page avec un hors-texte et une vignette en tête de chapitre gravés sur bois de Dignimont, Paris, Ferenczi, 1924 (Coll. « Le Livre moderne illustré »), 161 p., 20 cm, Bibliothèque nationale du Québec.

nom des illustrateurs sur la couverture illustrée puis sur la page titre. Cet usage signale l'importance de la recherche graphique, typographique et artistique. Aux Éditions Albert Lévesque, si cette pratique est généralisée dans tous les titres illustrés de la collection Les Poèmes, il en va autrement pour les autres séries. Dans Les Romans de la jeune génération, le nom de l'illustrateur est indiqué seulement en page-titre tandis que dans Les Romans historiques sa contribution n'est signalée qu'occasionnellement, peut-être selon sa renommée. Sans doute faut-il voir dans cette différence de traitement une conséquence liée au contexte de production des produits et à leur cible: Les Romans historiques, en particulier, sont destinés à un 
public jeune supposé moins sensible aux considérations artistiques inhérentes à la réalisation de l'objet. Toutefois, les éditeurs n'hésitent pas à faire appel à des artistes de qualité et de renom. Si du coté français les artistes Émile Beaume (18881967), Jean Renefer (1879-1957), Guy Arnoux (1886-1951) (lllustration 7) et Jean Lébédeff (1884-1970) comptent parmi les plus fameux graveurs et illustrateurs de l'époque, les Éditions Albert Lévesque bénéficient des contributions remarquables d'Adrien Hébert, de Rodolphe Duguay, d'Henri Beaulac ou de Simone Hudon. Ces collaborations révèlent un souci artistique commun à ces trois maisons d'édition. Chaque livre suppose la participation d'un illustrateur différent et, si l'auteur participe à ce choix, l'éditeur, dans son nouveau rôle de médiateur de la création artistique et de conseiller privilégié, intervient grandement dans cette décision. Il n'existe d'ailleurs pas un illustrateur qui soit attitré à l'une de ces cinq collections. Cet usage est pourtant répandu chez de nombreux éditeurs québécois comme Beauchemin avec Henri Julien (1852-1908), les Éditions Édouard Garand avec Albert Fournier ou les Éditions Lucien Parizeau avec Robert La Palme ${ }^{59}$. On remarque enfin, chez les deux éditeurs parisiens, que les illustrateurs Jean Renefer pour Fayard et Clément Serveau (1886-1972) pour Ferenczi ont réalisé des spécimens de couverture qui permettent au lecteur d'identifier instantanément chaque ouvrage et sa collection. Si l'on constate que les couvertures en couleurs réalisées par le graveur Ivan Jobin favorisent une harmonie dans la mise en forme extérieure de la collection Les Romans bistoriques, cette pratique ne se vérifie ni dans Les Romans de la jeune génération (où les couvertures ne sont pas illustrées) ni dans Les Poèmes (où les couvertures diffèrent d'un titre à l'autre).

Comme l'a souligné Silvie Bernier, il semble possible de remarquer aux Éditions Albert Lévesque les premiers symptômes d'une « coalition des avant-gardes ${ }^{60} »$. Ce phénomène a 


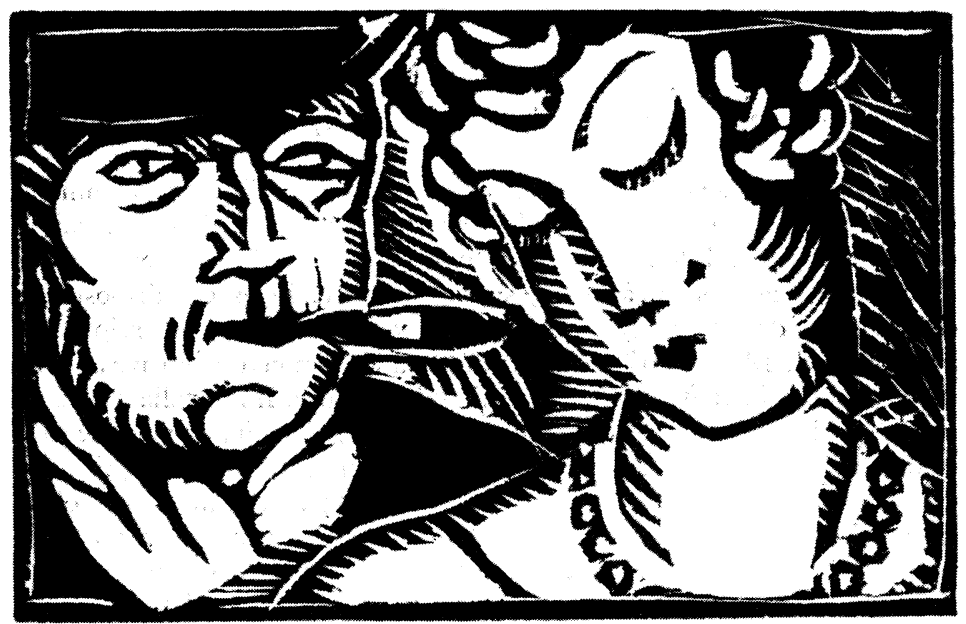

Illustration 7 : Henri Duvernois, Maxime, avec une vignette en tête de chapitre gravée sur bois de Guy Arnoux, Paris, Fayard, 1927 (Coll. « Le Livre de Demain »), 125 p., $24 \mathrm{~cm}$, Bibliothèque nationale du Québec.

pris naissance au sein du livre d'artiste français à la fin du XIX ${ }^{\mathrm{e}}$ siècle, en réaction à l'asservissement de l'image au texte. Il s'est d'abord manifesté dans les ouvrages de poésie contemporaine $^{61}$ puis s'est peu à peu répandu à l'ensemble de la discipline de l'illustration. Les collections Le Livre de demain et Le Livre moderne illustré de Fayard et de Ferenczi sont héritières de cette conception d'un rapport nouveau entre le contenu narratif et le contenu plastique basé sur une certaine autonomie des pratiques et surtout un refus de l'image descriptive. Or, « dans le Québec des années trente commence à se manifester une certaine sympathie entre producteurs artistiques et producteurs littéraires de la jeune génération ${ }^{62}$ ». Les plasticiens et les écrivains québécois commencent à prôner une certaine indépendance des procédés ainsi que l'importance de l'expérimentation plastique et linguistique. Ce discours, qui constitue une rupture avec l'idéologie dominante, favorise une modification de la fonction illustrative : "Plutôt 
que de donner un rendu servile du texte, l'image est conçue comme une cuvre d'art, à la limite viable sans le support de l'écrit ${ }^{63} \%$. Ainsi, en opposition avec l'organisation très stricte des illustrations de la populaire collection Les Romans canadiens, publiée entre 1923 et 1931 par les Éditions Édouard Garand et inspirée de la Select-Collection lancée en 1914 par l'éditeur parisien Ernest Flammarion, nous constatons aux Éditions Albert Lévesque plusieurs propriétés caractérisant ce nouveau rapport ${ }^{64}$. Les légendes disparaissent totalement (Le Dilettante) ou se contentent de résumer une idée ou de présenter un personnage (Dans les Ombres). La représentation de protagonistes et de scènes au contenu explicite diminue au profit des paysages (Dominantes) ou des natures-mortes $(D u$ Soleil sur l'étang noir). Enfin, les compositions se caractérisent par une stylisation de plus en plus manifeste qui reflète l'état des recherches plastiques de l'époque. Les artistes disposent manifestement d'une plus grande autonomie. Tandis que les séries Le Livre de demain et Le Livre moderne illustré constituent des collections très nettement identifiées comme telles (les couvertures et l'organisation de l'ornementation sont partout identiques), cette caractéristique s'applique moins aux publications des Éditions Albert Lévesque. Cette situation est encore plus manifeste dans la collection Les Poèmes, caractérisée d'un titre à l'autre par un manque d'harmonie dans les mises en page et par une certaine variété des médiums et des intervenants, autant d'éléments possiblement révélateurs d'une plus grande liberté accordée aux illustrateurs et aux écrivains.

\section{$* * *$}

Partageant la volonté de quelques éditeurs français de proposer des textes de qualité et des publications simultanément esthétiques, attrayantes et accessibles au plus grand nombre, Albert Lévesque souhaite également consolider son 
statut d'éditeur culturel moderne. Cet objectif semble d'autant plus explicite qu'il conjugue un souci de développement de la littérature nationale à une quête de créations originales. Producteur de nombreuses nouveautés canadiennes-françaises, son rôle est essentiel pour comprendre l'essor de l'illustration du livre puisqu'un tiers de son imposant catalogue est accompagné d'images. Au cours de cette période, seules les Éditions Édouard Garand enregistrent une activité similaire, essentiellement grâce aux 80 ouvrages illustrés de la collection Les Romans canadiens. L'association de ce nationalisme soucieux de modernité à une forte influence étrangère dans la mise en forme des ouvrages peut sembler contradictoire. Il convient toutefois de ne pas percevoir les collections diffusées par Albert Lévesque comme de simples copies d'un concept emprunté à quelques collègues français. Des différences demeurent entre les collections françaises et québécoises et chaque emprunt est adapté aux besoins et à la sensibilité des lecteurs de la province. Si la diffusion de l'illustration française dans le nord de l'Amérique dépend de facteurs éditoriaux, elle découle également de l'influence des courants artistiques internationaux, du déplacement des artistes vers l'Europe, en particulier vers Paris, et de leur formation professionnelle. Ces axes de recherche permettent d'envisager la question des transferts culturels entre la France et le Québec sous l'angle privilégié de la formation des artistes et du rôle de l'éditeur dans la réalisation de l'artefact constitué par le livre illustré. L'entre-deux-guerres constitue en effet une période très riche où l'on voit se multiplier, essentiellement à Montréal, des expériences intellectuelles, littéraires, artistiques et éditoriales qui se démarquent progressivement du poids des traditions académiques et des tendances conservatrices, autant de circonstances qui concourent à l'avènement de la sphère de production des imprimés illustrés. De cette promo- 
tion du travail des illustrateurs, le livre gagne en valeur artistique. Si ce phénomène se développe au Québec avec les éditeurs de poésie dont la maison Erta sera l'une des expressions les plus achevées, la production générale du livre illustré subit à partir des années 1940 une baisse importante. Au Québec comme en France, les illustrateurs seront principalement cantonnés au secteur jeunesse. De son coté, Albert Lévesque, trop soucieux de s'ouvrir au grand public et de devancer les désirs de son lectorat, ne tint pas compte des véritables besoins du marché. En 1937, après onze années d'efforts et en proie à de grosses difficultés financières, il vend son entreprise.

\section{NOTES}

${ }^{1}$ Jean-René Ostiguy, «Le livre illustré au Québec et en France (1900-1950) », Journal, Galerie nationale du Canada, $\mathrm{n}^{\circ} 43$ (septembre 1983), pp. 1-8.

${ }^{2} \mathrm{La}$ conception anglaise du livre illustré moderne s'inspire principalement des théories de William Morris (1834-1896), l'un des fondateurs du mouvement Arts and Crafts, précurseur du style Art Nouveau. Nostalgique de l'époque préindustrielle, Morris prône, dans la fabrication du livre, l'utilisation des meilleurs matériaux, la collaboration des meilleurs techniciens et une conception graphique impeccable. Le livre devient un objet esthétique rare et précieux mais, à la différence des bibliophiles et artistes français, l'illustration n'occupe pour lui qu'une fonction décorative et secondaire, souvent inspirée des motifs médiévaux.

${ }^{3}$ Le terme de « vignette » désigne divers ornements employés en typographie. Le bandeau est un ornement décoratif en forme de bande plus ou moins étroite qui se place en tête de la page de départ d'un livre, d'un chapitre ou d'une partie (il s'agit souvent d'une frise). Le cul-de-lampe est un motif décoratif typographique ou gravé placé dans le blanc des fins d'un chapitre. Armand Israël. "Glossaire », dans Armand Israël, dir. Livres d'art : histoire et techniques, Lausanne, Bibliothèque cantonale et universitaire/Paris, Éditions des Catalogues Raisonnés, 1994, pp. 213, 203 et 205. L'en-tête est une vignette placée au-dessus d'un chapitre, dans la partie supérieure de la page. 
Paul Robert, "En-tête », Le Petit Robert. Dictionnaire alphabétique et analogique de la langue française, Paris, Les Dictionnaires Le Robert, 1989, vol. 1, p. 655.

${ }^{4}$ François Landry, Beauchemin et l'édition au Québec. Une culture modèle, 18401940, Montréal, Fides, 1997, p. 81.

${ }^{5}$ Les éditeurs belges et suisses ont développé très tôt un champ éditorial en exploitant les lacunes du marché français limitrophe. Ils exportent également vers le Québec.

${ }^{6} \mathrm{Au}$ milieu du XIX' siècle, la France, dont la production est supérieure aux besoins de sa population, exporte déjà sept fois plus d'ouvrages qu'elle n'en importe, particulièrement vers les pays francophones d'Europe et d'Amérique. Bruno Blasselle, Histoire du livre. Le triomphe de l'édition, Paris, Gallimard, 1997, tome 2, p. 33. (Coll. «Découvertes Gallimard Histoire »).

${ }^{7}$ Jacques Michon, «Édition littéraire et autonomie culturelle, le cas du Québec », Présence francophone, no 26 (1985), pp. 58-59.

${ }^{8}$ Michel Verrette, L'alphabétisation au Québec. 1600-1900. En marche vers la modernité culturelle, Sillery, Septentrion, 2002, pp. 92-93.

'Yvan Lamonde, La librairie et l'édition à Montréal, 1776-1920, Montréal, Bibliothèque Nationale du Québec, 1991, p. 92.

${ }^{11}$ Entre 1886 et 1902, la maison Beauchemin enregistre une production composée à $14 \%$ de manuels scolaires et à $9 \%$ d'ouvrages littéraires. Entre 1902 et 1939 , ces chiffres passent à $24 \%$ et $22 \%$ avec en outre $6 \%$ consacrée à l'édition de poésie. Landry, op. cit., p. 222.

${ }^{11}$ Silvie Bernier, Du texte à l'image. Le livre illustré au Québec, Sainte-Foy, Presses de l’Université Laval, 1990, pp. 65-67. (Coll. «Vie des Lettres québécoises »).

${ }^{12}$ La première édition du roman Maria Chapdelaine, plutôt pauvre matériellement, en constitue un bon exemple. Voir Louis Hémon, Maria Chapdelaine. Récit du Canada français, avec vingt-cinq dessins au fusain de Suzor-Coté, Montréal, Lefebvre, 1916, xix-243 p.

${ }^{13}$ Bernier, op. cit., p. 74.

${ }^{14}$ Richard Giguère et al., "L'éditeur littéraire professionnel », dans Jacques Michon, dir. Histoire de l'édition littéraire an Québec au XX' siècle. La Naissance de l'éditeur, 1900-1939, Montréal, Fides, 1999, vol. 1, pp. 279-280.

${ }^{15}$ Ibid., p. 21.

${ }^{16}$ L'association La Ligue des droits du français, fondée en 1913, se donne pour mission de rehausser la qualité du français et d'en rétablir l'usage dans 
certains secteurs de la vie sociale. Elle soutient la publication de plusieurs ouvrages de romanciers canadiens-français ainsi qu'une revue, L'Action française. En 1918, la Ligue se dote d'un département d'édition en fondant la Bibliothèque de l'Action française puis, en 1919, d'un magasin appelé la Librairie de l'Action française qui diffuse les ouvrages du catalogue de la Bibliothèque d'Action française ainsi que certains auteurs canadiens et étrangers. En 1921, la Ligue des droits du français devient la Ligue d'Action française, ce qui témoigne de l'élargissement de son œuvre. L'abbé Lionel Groulx en sera le directeur de 1918 à 1926. Il prend officiellement parti pour une littérature nationale à la fois française, canadienne et catholique. Pierre Hébert et Dominique Garand, "L'édition nationaliste saisie par la crise », dans Jacques Michon, dir. Histoire de l'édition littéraire an Québec an XX' siècle. La Naissance de l'éditeur, 1900-1939, Montréal, Fides, 1999, vol. 1, pp. 252-263.

${ }^{17}$ À la fin de l'année 1925, Lévesque découvre que Victor Hermann, le gérant de la librairie, et son complice ont fondé une compagnie parallèle qui écoule des livres achetés par la Librairie de l'Action française pour en détourner les profits. Ibid., p. 262. Voir aussi Albert Lévesque, « Rectification aux Mémoires de Lionel Groulx », dans Jacques Michon, dir. L'édition littéraire en quête d'autonomie : Albert Lévesque et son temps, Sainte-Foy, Presses de l'Université Laval, 1994, pp. 116-117.

${ }^{18}$ En juin 1925, Lévesque avait épousé une des filles de Joseph-Onésime Labrecque, riche commerçant de charbon et principal actionnaire de la Banque Nationale. Labrecque était connu pour sa sympathie à l'égard du mouvement nationaliste. Hébert et Garand, loc. cit., p. 262.

${ }^{19}$ Ibid., pp. 262-263. Cet accord, d'une durée de trois années, portait sur l'appellation de la société mais permettait également aux anciens directeurs de la Ligue de publier librement la revue mensuelle et l'almanach annuel. Ceuxci conservaient de plus un droit de regard sur les publications de la nouvelle maison d'édition. Toutes ces précautions semblent entrer dans une volonté de camoufler le changement de direction et les scandales financiers de l'entreprise.

${ }^{20}$ Liette Bergeron, " Catalogue de la Librairie d'Action canadienne-française et des Éditions Albert Lévesque », dans Jacques Michon, dir. L'édition littéraire en quête d'autonomie : Albert Lévesque et son temps, Sainte-Foy, Presses de l'Université Laval, 1994, vol. 1, pp. 165-200.

${ }^{21}$ Landry, op. cit., pp. 97-99.

${ }^{22}$ Jacques Michon et Josée Vincent, "Le libraire-éditeur », dans Jacques Michon, dir. Histoire de l'édition littéraire au Québec au XX' siècle. La Naissance de l'éditeur, 
1900-1939, Montréal, Fides, 1999, vol. 1, pp. 103 et 418-419 (annexe 6). Cornélius Déom (1863-1946) se rendait d'ailleurs chaque année « en Europe pour négocier directement avec ses fournisseurs belges et français » (p. 418).

${ }^{23}$ Lucie Robert, « Prolégomènes à une étude sur les transformations du marché du livre au Québec (1900-1940) ", dans Yvan Lamonde, dir. L'imprimé au Québec. Aspects historiques (18-20 siècles), Québec, IQRC, 1983, p. 232. (Coll. "Culture savante »).

${ }^{24}$ Jean-René Ostiguy signale que les bibliothèques publiques s'en départissent facilement à l'occasion des ventes annuelles. Ostiguy, loc. cit., p. 3.

${ }^{25}$ Isabelle de Conihout, «La conjoncture de l'édition », dans Roger Chartier et Henri-Jean Martin, dir. Histoire de l'édition française. Le livre concurrencé : 19001950, Paris, Fayard/Cercle de la Librairie, 1989 [1983], vol. 4, p. 85.

${ }^{26} \mathrm{Il}$ faut naturellement distinguer les différents tirages d'un même ouvrage : il n'est pas rare qu'un livre, particulièrement quand il est illustré, bénéficie d'un premier tirage limité à quelques dizaines d'exemplaires sur un papier plus luxueux. Aux Éditions Albert Lévesque, on retrouve cette pratique pour certains recueils de poésies comme Aux Sources claires ou Du Soleil sur l'étang noir. D'autres distinctions peuvent ensuite être induites selon que ces éditions soient numérotées ou non, tirées à la main ou la presse, etc. Ces manipulations ont une incidence sur le prix de l'ouvrage qui est destiné soit à un public spécialisé de bibliophiles soit au lecteur moyen. Notons que les références consultées dans le cadre de cette étude sont toutes des réalisations destinées au grand public.

${ }^{27}$ Aujourd'hui, on retrouve ces ouvrages dans les collections patrimoniales de la Bibliothèque nationale du Québec ainsi qu'au sein de diverses bibliothèques universitaires telles que l'Université de Montréal et l'Université du Québec à Montréal.

${ }^{28}$ Ostiguy, loc. cit., p. 6.

${ }^{29}$ Suzanne Pouliot et Jacques Michon, «Les éditeurs pour la jeunesse », dans Jacques Michon, dir. Histoire de l'édition littéraire au Québec au XX' siècle. La Naissance de l'éditeur, 1900-1939, Montréal, Fides, 1999, vol. 1, pp. 375-376.

${ }^{30}$ En 1935, lorsque Harry Bernard (1898-1979) publie Montcalm se fáche chez Lévesque, il a déjà reçu trois fois le Prix David (en 1924, 1925 et 1931). Réginald Hamel, John Hare et Paul Wyczynski, Dictionnaire pratique des auteurs québécois, Montréal, Fides, 1976, pp. 57-58.

${ }^{31}$ Lorsqu'elle publie La Sorcière de l'âlot noir dans Les Romans historiques aux Éditions Albert Lévesque, Marie-Antoinette Grégoire-Coupal (1905-1984) a 
déjà remporté le Prix d'Action intellectuelle de 1932 pour Le Sanglot sous les rires (également paru chez Lévesque). Elle reçoit d'ailleurs, pour La Sorcière de l'ìlot noir, la médaille d'or de l'Action française. Marc-André Goulet et François Landry, "L'édition populaire : Édouard Garand ", dans Jacques Michon, dir. Histoire de l'édition littéraire au Québec au XX' siècle. La Naissance de l'éditeur, 1900-1939, Montréal, Fides, 1999, vol. 1, pp. 318-319.

${ }^{32}$ Maxine est le pseudonyme de Marie-Caroline-Alexandra Taschereau-Fortier (1874-1957). Après avoir travaillé comme traductrice à la Gazette officielle de l'Assemblée législative du Québec, elle entame tardivement une carrière dans l'écriture. Au cours des années 1920 et 1930, elle travaille principalement pour les Éditions Albert Lévesque. Par la suite, elle publie de nombreux titres dans les collections Histoire du Canada pour les tout-petits (1946) et La petite Histoire (1957) aux Éditions Beauchemin. Très prolifique, elle est l'auteure de plus de trente livres destinés aux enfants. On lui connaît également deux romans pour adultes publiés au début des années 1930. Françoise Lepage, Histoire de la littérature pour la jeunesse (Québec et francophonies du Canada) suivi d'un Dictionnaire des auteurs et des illustrateurs, Orléans, Les Éditions David, 2000, pp. 685686.

${ }^{33}$ Notons que cet ouvrage accompagné des dessins de Lemieux a fait l'objet d'une première publication en 1930 au sein de la collection Les Récompenses, également destinée aux adolescents.

${ }^{34}$ De 1927 à 1936, le secteur jeunesse représente en effet $31 \%$ des activités des Éditions Albert Lévesque. Giguère et al., loc. cit., p. 286.

${ }^{35}$ Jacques Michon, «Albert Lévesque, entre « individualistes » et nationalistes ", dans Jacques Michon, dir. L'édition littéraire en quête d'autonomie : Albert Lévesque et son temps, Sainte-Foy, Presses de l'Université Laval, 1994, pp. 103.

${ }^{36}$ Giguère et al., loc. cit., p. 289.

${ }^{37}$ Nérée Beauchemin et Louis Dantin représentent également l'ancienne génération au sein de cette collection tandis qu'Alfred DesRochers et Robert Choquette sont parmi les quelques « jeunes » auteurs.

${ }^{38}$ Voir par exemple l'état de la collection ("Dans la même série / "Les Poìmes" / ... ») sur le plat (carton formant la couverture) verso du recueil Les Masques déchirés de Jovette-Alice Bernier ainsi que le sigle en page titre.

${ }^{39}$ Gérard Genette, Seuils, Paris, Seuil, 1987, p. 27. (Coll. « Points Essai »).

${ }^{40}$ Jacques Michon, «L'édition littéraire au Québec de 1940 à 1960 », Itinéraires et Contacts de Cultures, vol. 6 (1985), p. 63. 
${ }^{41}$ Denis Martin, L'estampe au Québec, 1900-1950, Québec, Musée du Québec, 28 avril-3 juillet 1988, Québec, Musée du Québec, 1988, p. 21.

${ }^{42}$ Rodolphe Duguay, Carnets intimes, présenté par Hervé Biron, Montréal, Boréal Express, 1978.

${ }^{43}$ Dennis Reid, Edwin H. Holgate, Ottawa, Galerie nationale du Canada, 1976, pp. 7-9. (Coll. « Artistes canadiens, $\left.{ }^{\circ} 4 »\right)$.

${ }^{44}$ Duguay, op. cit., pp. 34 et 187.

${ }^{45}$ Martin, op. cit., p. 70.

${ }^{40}$ Maurice Busset, La Technique moderne du bois gravé, Paris, Delagrave, 1925, Morin-Jean, Manuel pratique du graveur sur bois, Paris, Henri Laurens, 1926 et Charles Saunier, Les Décorateurs du livre, Paris, F. Rieder et Cie Éditeurs, 1922. (Coll. « L'art français depuis vingt ans »).

${ }^{47}$ Denise A. Ostiguy, "L'illustration du livre au Québec », dans George A. Chartrand, dir. Livres, bibliothèques et culture québécoise, mélanges offerts à Edmond Desrochers, Montréal, ASTED, 1977, vol. 1, p. 103.

${ }^{48}$ Georges Bouchard, Other Days, Other Ways, avec 25 bois gravés d'Edwin $\mathrm{H}$. Holgate, Montréal/New York, Louis Carrier et Cie/The Mercury Press, 1928, 189 p., $21 \mathrm{~cm}$ (édition en français en 1929 sous le titre Vieilles choses, vieilles gens) et Robert Choquette, Metropolitan Museum, avec 13 bois gravés d'Edwin H. Holgate, Montréal, sur les presses d'Herald Press pour le compte des auteurs, 1931,29 p., $30 \mathrm{~cm}$.

${ }^{49}$ Holgate enseigne à l'École des beaux-arts de Montréal jusqu'en 1934.

${ }^{50}$ Pilot est également de ceux qui ont étudié à Paris au début des années 1920. Tout comme ses amis Holgate, Duguay et Adrien Hébert, il s'initie à la gravure en autodidacte. Martin, op. cit., p. 22.

${ }^{51}$ Ostiguy, loc. cit., pp. 6-7. Ostiguy appuie son propos sur le catalogue du Premier grand Salon des Anciens des Beaux-arts de Montréal, tenu en 1925, qui fait mention d'une autre exposition, organisée par l'École et plusieurs librairies montréalaises et présentant de nombreux livres illustrés français.

${ }^{52}$ Daniel Renoult, «Les nouvelles possibilités techniques : le triomphe de la mécanique ", dans Roger Chartier et Henri-Jean Martin, dir. Histoire de l'édition française. Le livre concurrencé: 1900-1950, Paris, Fayard/Cercle de la Librairie, 1989 [1983], vol. 4, p. 38. 
${ }^{53}$ Dans les collections à l'étude, ces chiffres tiennent compte à la fois des illustrations hors-texte et in-texte, incluant les vignettes, les têtes de chapitres et les culs-de-lampe.

${ }^{54}$ Michon, loc. cit. (1994), pp. 105 et 112.

${ }^{55}$ Pierre Landry, L'apport de l'art Nouveau aux arts graphiques au Québec de 1898 à 1910, thèse de maîtrise (histoire de l'art), Université Laval, 1983, p. 111.

${ }^{56}$ Daniel Renoult, «La mise en page », dans Roger Chartier et Henri-Jean Martin, dir. Histoire de l'édition frangaise. Le livre concurrencé : 1900-1950, Paris, Fayard/Cercle de la Librairie, 1989 [1983], vol. 4, p. 403.

${ }^{57}$ Il est en effet possible de constater la présence de rares lettrines ou vignettes in-texte de petit format chez Fayard, comme dans Civilisation. 1914-1917 de Georges Duhamel, publié en 1927 dans la collection Le Livre de demain et orné de cinquante bois originaux de Raymond Thiollière.

${ }^{58}$ Renoult, loc. cit., p. 403.

${ }^{59}$ Bernier, op. cit., pp. 79-80.

${ }^{60}$ Ibid., pp. 80-81.

${ }^{61}$ Pensons au célèbre exemple de l'édition de Parallèlement de Paul Verlaine, édité en 1900 par Ambroise Vollard et accompagné des lithographies originales de Pierre Bonnard.

${ }^{62}$ Bernier, op. cit., p. 80.

${ }^{63}$ Ibid.

${ }^{64}$ Il faut faire exception de la collection Les Romans historiques : puisqu'il s'agit d'ouvrages destinés à la jeunesse, les illustrations, sans constituer nécessairement une paraphrase du texte, entretiennent des liens plus étroits avec lui, comme en témoigne la présence systématique des légendes. Toutefois, il est possible de relever, au sein de certaines références de cette série, une stylisation marquée qui traduit un souci de s'attacher d'avantage à la qualité de la composition plastique qu'à la représentation du contenu narratif. Nous pouvons citer l'exemple des dessins réalisés par Jean-Paul Lemieux pour Le petit Page de Frontenac ainsi que celui des linogravures d'Henri Beaulac pour Montcalm se fâche. 